\title{
2D Quasi Exact Inverse of PA model in Digital Predistorter for Concurrent Dual-Band System
}

\author{
Naveen Naraharisetti*, Patrick Roblin*, Christophe Quindroit*, Meenakshi Rawat*, Shahin Gheitanchi ${ }^{\dagger}$ \\ *Ohio State University, Department of Electrical \& Computer Engineering, Columbus, OH, 43210, USA \\ ${ }^{\dagger}$ Altera Europe Limited, High Wycombe, Buckinghamshire, HP12 4XF, England
}

\begin{abstract}
This paper reports a new direct learning (DL) technique using 2D quasi exact inverse (2D-QEI) of a power amplifier (PA) model for linearizing concurrent dual band PA. In contrast to indirect learning (IL) architecture, where the coefficients are extracted by swapping the input and output signals in any PA model, a QEI of a PA model can be used in the digital predistorter (DPD). A 2D memory polynomial (2D-MP) is used in both the cases to compare the performance. The evaluation of the model's performance is conducted on an application close to real base station using a field programmable gate array (FPGA) and two radio transceivers. A $10 \mathrm{~W} P A$ is excited with two wideband code division multiple access (WCDMA) signals of $3.84 \mathrm{MHz}$ bandwidth which are $310 \mathrm{MHz}$ apart. The measurement results demonstrate that in the presence of additive noise, there is a noticeable improvement in terms of normalized mean square error (NMSE) and adjacent channel power ratio (ACPR) when using the QEI model for DPD. This improvement is achieved in a single step with no iteration as in practical DPD systems.

Index Terms-power amplifier, digital predistortion, linearization, direct learning, in-direct learning.
\end{abstract}

\section{INTRODUCTION}

High spectral efficient modulation techniques like WCDMA and orthogonal frequency division multiplexing (OFDM) have non-constant envelop with high peak to average power ratio (PAPR). Although it imposes a strict linearity requirement within the system, the inherent non-linearity of the power amplifier(PA) develops spectral regrowth in in-band and out of band. DPD is one of the commonly used linearizing techniques because of its robustness, moderate implementation cost and high accuracy.

A broadband PA for base station has become available to handle widely separated carriers for concurrent dual band transmission. Out of band intermodulation distortion (IMD) products can be easily filtered out whereas DPD can be used for the in-band linearization. The sampling frequency requirements for analog to digital(ADC) and digital to analog(DAC) converters are greatly reduced by implementing DPD using frequency selective technique as in [1], [2].

The generalized architecture of a dual band transmitter using Frequency selective technique is shown in Fig. 1. Most of the available DPD's are based on IL as shown in Fig. 2(a), wherein the inverse of the PA is modeled using a postdistorter inverse model and the coefficients are transferred to the PD. The two main drawbacks that affect the performance of this method are [3]: 1) Due to the measurement noise at the output, IL requires to find an inversion of the noisy regression matrix. Hence, the adaptive algorithm converges to biased values. 2) Commutative property is assumed for non-linear systems.

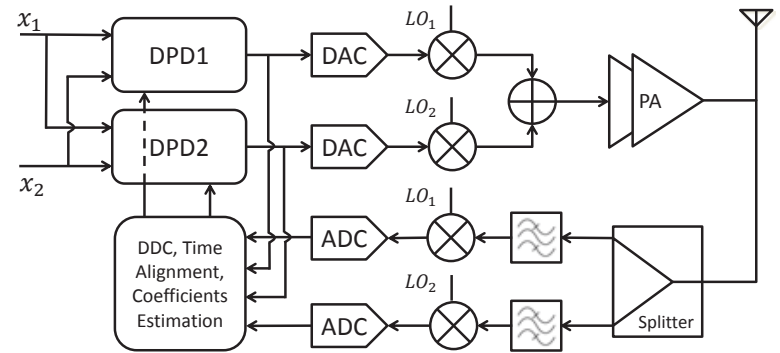

Fig. 1. Block Diagram of Test Bench

The DL technique is developed by extracting an accurate PA model (PAM) initially, and then the DPD function is obtained by inverting the PA model. The architecture used for DL is shown in Fig. 2(b), which is extended version of single band DL architecture [4] for dual-band DL system. A new DL technique using the quasi-exact inverse of the PA model is developed for single band case in [4], [5], showing performance improvement in terms of ACPR and NMSE when compared to IL. In this paper, the same principle is extended to dual-band case to incorporate these benefits into the DPD system. This is the first DL technique developed for dual band linearization to the best of author's knowledge. The paper is organized as follows: The principle of IL is explained in Section II, and a 2D quasi exact inverse (2D-QEI) model for DL architecture is developed in Section III. The 2D-QEI model is verified using a FPGA setup, which is close to the real base station, is described in Section IV. Finally the paper is concluded in Section V by comparing the performance of both the methods.

\section{INDIRECT LEARNING}

The IL architecture shown in Fig.2(a) is commonly used for identifying the predistorter. The PA is preceded by a predistorter and followed by a post-distorter. This algorithm uses two similar memory polynomial (MP) models for both the post-distorter and PD [2]. The DPD is implemented in two steps.

1) In the initial training, when there is no PD attached, the scaled version of output is provided as the input to the post-distorter. The coefficients are extracted using the least squares (LS) method in order to reduce the error $e$. Ideally, if we can reach $e \simeq 0$ then $\tilde{z} \simeq z$, creating the post-inverse for the PA. 


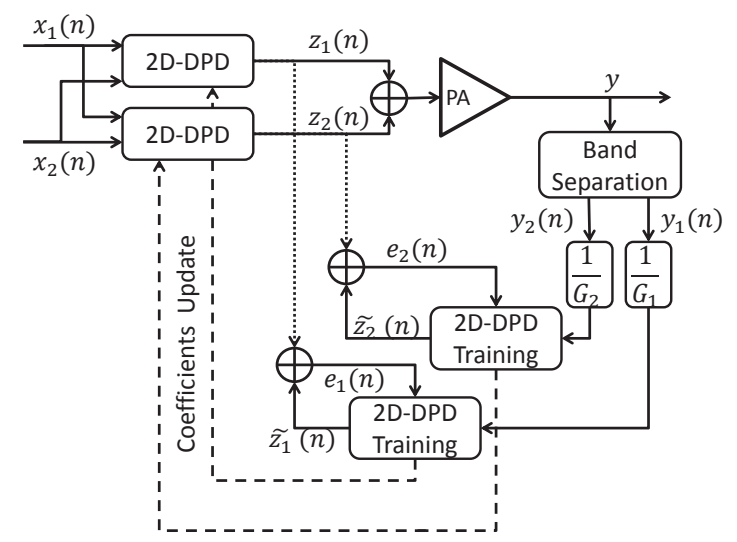

(a) In-Direct Learning

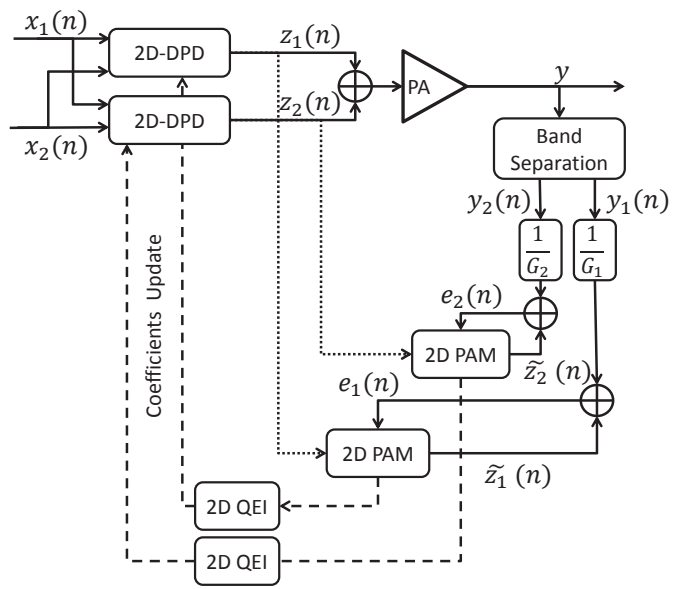

(b) Quasi-Exact Inverse

Fig. 2. Different Learning Schemes

2) Next, these coefficients are copied into the DPD, which is used as pre-inverse.

The MP model used in this algorithm is defined in (1) where we include only the odd order terms [2]:

$$
z_{i}(n)=\sum_{m=0}^{M-1} \sum_{k=0}^{N-1} \sum_{j=0}^{k} c_{j k m}^{(i)}\left|x_{i}(n-m)\right|^{2 k}\left|x_{l}(n-m)\right|^{2(k-j)} \times
$$

where $\forall i, l \in[1,2]$ and $i \neq l, x_{i}(n-m)$ is the delayed input, $K$ is the non-linearity order, $M$ is the memory depth, $c_{j k m}^{(i)}$ are the coefficients of the model and $z_{i}(n)$ is the predistorted output from the PD.

\section{2D QUASI EXACT INVERSE}

A generalized memory PA model can be given as:

$y_{i}(n)=\sum_{m_{a}=0}^{M_{A}-1} G_{i, m_{a}}\left(\left|z_{i}\left(n-m_{a}\right)\right|^{2},\left|z_{l}\left(n-m_{a}\right)\right|^{2}\right) z_{i}\left(n-m_{a}\right)$

where $\left|z_{i}\left(n-m_{a}\right)\right|^{2}$ is envelop squares of each band with memory, $G_{1, m_{a}}($.$) and G_{2, m_{a}}($.$) are the gain functions for$ each band. The gain functions with memory are functions of the envelop squares of each band. The gains $G_{i, m_{a}}$, can be implemented using memory polynomial, splines or any other functions. In order to compare the performance between the DL and IL in the presence of noise, 2D memory polynomials are used here.

The DPD output can be written as given in (3):

$$
z_{i}(n)=\sum_{m_{p}=0}^{M_{P}-1} \zeta_{i, m_{p}}(n) x_{i}\left(n-m_{p}\right)
$$

where $M_{P}$ is the DPD memory depth and $\zeta_{i, m_{p}}$ are the gain of the predistorters for each memory depth.
Substituting (3) in (2) gives the overall equation for the cascaded system (PA and DPD):

$$
\begin{gathered}
y_{i}(n)=\sum_{m_{a}=0}^{M_{A}-1} \sum_{m_{p}=0}^{M_{P}-1} G_{i, m_{a}}\left(\left|z_{i}\left(n-m_{a}\right)\right|^{2},\left|z_{l}\left(n-m_{a}\right)\right|^{2}\right) \times \\
\zeta_{i, m_{p}}\left(n-m_{a}\right) x_{i}\left(n-m_{a}-m_{p}\right)
\end{gathered}
$$

On setting all the coefficients weighing $x_{i}\left(n-m_{a}-m_{p}\right)$ to zero, except for the case $m_{a}+m_{p}=m_{a}=m_{p}=0$, which is the linear gain of $\alpha_{i} G_{i, A}^{l i n}$ with $\alpha_{i}$ as the targeted gain compression verifying $0<\alpha_{i}<1$. Hence (4) reduces to the following system of equations:

$$
\begin{gathered}
\sum_{m_{a}=\max \left(0, k-M_{P}\right)}^{\min \left(k, M_{A}\right)} G_{i, m_{a}}\left(\left|z_{1}\left(n-m_{a}\right)\right|^{2},\left|z_{2}\left(n-m_{a}\right)\right|^{2}\right) \times \\
\zeta_{i, k-m_{a}}\left(n-m_{a}\right)=\alpha_{i} G_{i, A}^{l i n} \delta_{k}, \quad \forall 0 \leq k \leq M_{A}+M_{P}
\end{gathered}
$$

The linearization of PA model with $M_{A}+1$ taps leads to a system of $M_{A}+M_{P}+1$ equations with $M_{P}+1$ unknown predistorter coefficients. Since this is an overdetermined system, the $M_{P}+1$ equations are used to extract the coefficients whereas the remaining introduce the residual error. This residual error can be reduced with an increase in DPD memory depth $M_{P}$. Ideally an exact solution is found when this $M_{P}$ approaches infinity. The general solution with arbitrary number of taps is:

$$
\zeta_{i, 0}(n)=\frac{\alpha_{i} G_{i, A}^{l i n}}{G_{i, 0}\left(\left|z_{1}(n)\right|^{2},\left|z_{2}(n)\right|^{2}\right)}
$$




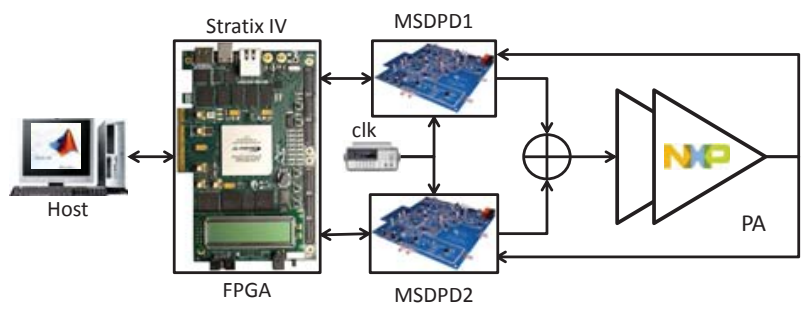

Fig. 3. Test Bench Setup

$$
\begin{aligned}
& \zeta_{i, k}(n)=-\frac{1}{G_{i, 0}\left(\left|z_{1}(n)\right|^{2},\left|z_{2}(n)\right|^{2}\right)} \\
& \sum_{m_{a}=\max \left(1, k-M_{P}\right)}^{\min \left(k, M_{A}\right)} G_{i, m_{a}}\left(\left|z_{1}\left(n-m_{a}\right)\right|^{2},\left|z_{1}\left(n-m_{a}\right)\right|^{2}\right) \times \\
& \zeta_{i, k-m_{a}}\left(n-m_{a}\right) \quad \forall 1 \leq k \leq M_{P}
\end{aligned}
$$

The solution for the memoryless case is given by (6), whereas the memory case is obtained from (7). The key feature in (6) and (7) is that the envelop $\left|z_{1}(n)\right|^{2}$ and $\left|z_{2}(n)\right|^{2}$ at the output of both the PD's are unknown at time $n$ and must be selfconsistently calculated at each new time step using (8).

$$
\begin{aligned}
& f_{i}\left(\left|z_{1}(n)\right|^{2},\left|z_{2}(n)\right|^{2}\right) \\
& \triangleq\left|G_{i, 0}\left(\left|z_{1}(n)\right|^{2},\left|z_{2}(n)\right|^{2}\right)\right|^{2}\left|z_{i}(n)\right|^{2} \\
&=\left|\sum_{m_{p}=0}^{M_{P}} G_{i, 0}\left(\left|z_{1}(n)\right|^{2},\left|z_{2}(n)\right|^{2}\right) \zeta_{i, m_{p}}(n) x_{i}\left(n-m_{p}\right)\right|^{2} \\
& \triangleq\left|S_{i}(n)\right|^{2}
\end{aligned}
$$

Since $S_{i}(n)$ is readily calculated from (6) and (7), the unknown envelops $\left\langle\left|z_{1}(n)\right|^{2},\left|z_{2}(n)\right|^{2}\right\rangle$ are obtained from the inverse of the zero delay PA model output $f_{i}^{-1}\left(|S(n)|^{2}\right)$. This implies that for the 2D-QEI to exist, each zero-delay AMAM PA outputs $\left[f_{1}\left(\left|z_{1}(n)\right|^{2},\left|z_{2}(n)\right|^{2}\right), f_{2}\left(\left|z_{1}(n)\right|^{2},\left|z_{2}(n)\right|^{2}\right]\right.$ must originate from a single input pair $\left[\left|z_{1}(n)\right|^{2},\left|z_{2}(n)\right|^{2}\right]$. This requirement is usually satisfied as long as the PA is not over-saturated.

\section{Measurement Results}

The test bench setup is shown in the Fig. 3. The input data is sent from a host computer using Matlab and stored in the FPGA's memory. In this case, a WCDMA signal of $3.84 \mathrm{MHz}$ bandwidth generated from different seeds is used in both the channels. The downloaded data can be played out to analog devices mixed signal DPD (ADI MSDPD) board at the rate of $245.76 \mathrm{MHz}$. An ADI MSDPD board integrates a complete high performance RF and mixed-signal transmit and receive chain onto a single board. The signal is upconverted using an I/Q modulator and sent to a $10 \mathrm{~W}$ NXP PA. The frequency spacing between the bands is around $310 \mathrm{MHz}$, where the lower band is transmitted at $1890 \mathrm{MHz}$ and the
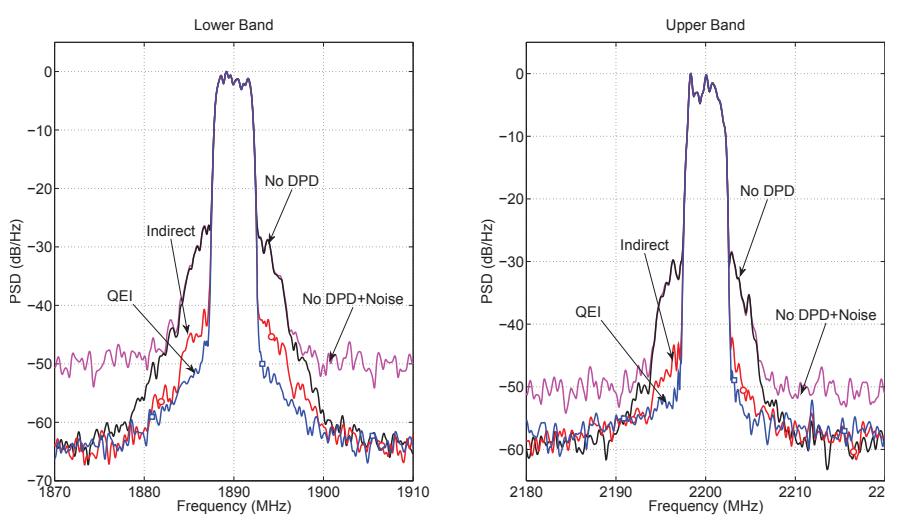

Fig. 4. Measurement Results: Spectrum

upper band is at $2200 \mathrm{MHz}$. The output from the PA is fed back to the observation path on both the boards. The signal is downconverted to an $184.32 \mathrm{MHz}$ IF signal and stored in the FPGA's memory. The stored data can be used for further signal processing like digital down conversion (DDC), time alignment and extracting the coefficients for the DPD system.

A 2D MP with non-linearity order $(\mathrm{N}=7)$ and memory length $(M=2)$ are used in both the DL and IL model extractions. $M_{P}=10$ is used for calculating the QEI. No iteration is used for both the DL and IL DPD extractions, which are performed in a single step like in practical systems. The spectra without and with DPD for additive white noise in the receiver yielding a $-50 \mathrm{dBc}$ noise floor, are shown in Fig. 4. It can be observed from Fig. 4 and Table I that in the presence of additive white noise, the direct learning architecture using QEI performs better than the indirect learning architecture in terms of NMSE and ACPR by up to $4 \mathrm{~dB}$.

TABLE I

COMPARISON OF NMSE AND ACPR FOR $-50 d B c$ NOISE FLOOR

\begin{tabular}{|c|c|c|c|c|}
\hline \multirow{2}{*}{ Configuration } & \multicolumn{2}{|c|}{ NMSE(dB) } & \multicolumn{2}{c|}{$\begin{array}{c}\text { ACPR }(\mathrm{dBc}) \\
@ \pm 5 \mathrm{MHz}\end{array}$} \\
\cline { 2 - 5 } & LSB & USB & LSB & USB \\
\hline Without DPD & -20.99 & -21.94 & $-30.67 /-32.02$ & $-32.35 /-33.81$ \\
\hline Indirect Learning & -34.43 & -33.69 & $-44.49 /-44.46$ & $-45.97 /-47.66$ \\
\hline QEI & -38.43 & -37.42 & $-50.02 /-51.88$ & $-50.33 /-51.26$ \\
\hline
\end{tabular}

\section{CONCLusion}

In this paper, we developed a new 2D quasi-exact inverse for PA model using direct learning architecture and memory polynomials. The performance is compared with an indirect learning architecture using 2D MP, in a testbed which is close to a real-time base station. The DPD extraction is performed in a single step with no iteration as in real system where fast adaptation is critical. It is observed that when using the same number of coefficients, there is a $4 \mathrm{~dB}$ improvement in both NMSE and ACPR in the presence of the additive uniform white noise. 


\section{ACKNOWLEDGMENT}

The authors would like to thank Analog Devices Inc., Wilmington, MA and NXP Semiconductors, Smithfield, RI, for donating the Mixed Signal Digital Pre-distortion System Boards (MSDPD) and PAs respectively which are used in this study. The authors would also thank Altera Corporation / Wireless Systems Solutions Group for their financial and technical support for this project and the donation of the Stratix IV FPGA. This work was also supported in parts by the National Science Foundation under grant ECS 1129013.

\section{REFERENCES}

[1] P. Roblin, S. Myoung, D. Chaillot, Y. Kim, A. Fathimulla, J. Strahler, and S. Bibyk, "Frequency-Selective Predistortion Linearization of RF Power Amplifiers," IEEE Transactions on Microwave Theory and Techniques, vol. 56, no. 1, pp. 65-76, 2008.

[2] S. Bassam, M. Helaoui, and F. Ghannouchi, "2-D Digital Predistortion (2-D-DPD) Architecture for Concurrent Dual-Band Transmitters," IEEE Transactions on Microwave Theory and Techniques, vol. 59, no. 10, pp. 2547-2553, 2011.

[3] D. Zhou and V. DeBrunner, "Novel Adaptive Nonlinear Predistorters Based on the Direct Learning Algorithm," Signal Processing, IEEE Transactions on, vol. 55, no. 1, pp. 120-133, 2007.

[4] N. Naraharisetti, P. Roblin, C. Quindroit, M. Rawat, and S. Gheitanchi, "Quasi-Exact Inverse PA Model for Digital Predistorter Linearization," in 2013 82nd ARFTG Microwave measurement conference, 2013.

[5] P. Roblin, Nonlinear RF Circuits and Nonlinear Vector Network Analyzers. Cambridge University Press, 2011. 